REVISTA ACADÉMICA ECO (2020) 22: 15-23

\title{
NEURONEGOCIACIONES: GESTIÓN DEL DOBLE CONOCIMIENTO
}

Artículo de reflexión

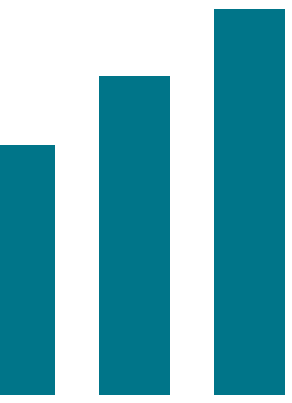

\section{Michael Eric Finkel Stern}

Magíster en Gestión de Conflictos y Estudios de Paz, Universidad Haifa, Israel.

Correo: mefinkel@correo.url.edu.gt

Fecha de recepción: 10 de noviembre de 2019

Fecha de aceptación: 29 de noviembre de 2019

\section{Resumen}

Las neurociencias han incursionado en nuevas áreas de aplicación y análisis. En los últimos años, campos relacionados a los negocios y al desarrollo personal han aplicado el estudio del sistema nervioso para desarrollar investigaciones, probar hipótesis y proponer metodologías para agilizar sus procesos, al utilizar el cerebro humano, sus comportamientos y reacciones para una gestión efectiva en la toma de decisiones. El campo de la negociación no se queda a un lado: las ciencias de los tiempos y la psicología han ampliado el horizonte de estudios para la gestión de conflictos y procesos de negociación, donde la comunicación y el conocimiento se tornan fundamentales para el éxito en estos campos de aplicación personal, profesional y social.

Palabras clave: neurociencias, negociación, psicología, comunicación, conocimiento, inteligencia emocional 


\title{
NEURO-NEGOTIATIONS: DOUBLE KNOWLEDGE MANAGEMENT
}

Article for reflection

\begin{abstract}
Neuroscience has made incursions into new areas of application and analysis. During recent years, human development and business related fields have applied the study of the nervous system to develop investigation, prove hypotheses, and propose methodologies in order to streamline processes by using human brain behaviors and reactions to upscale effective decision making. The field of negotiation has not been not left aside: contemporary sciences and psychology have widened the horizon in the study of conflict management and negotiation processes, where communication and knowledge are fundamental to achieve success in its personal, professional and social applications.
\end{abstract}

Key words: neuroscience, negotiation, psychology, communication, knowledge, emotional intelligence 


\section{Introducción}

La naturaleza del ser humano es social. Hacer énfasis en esta expresión conlleva al análisis de una serie de conceptos que resultan complejos al analizar su aplicación. Comunicación, conocimiento y negociación son términos que mantienen una aplicación genérica en la sociedad, pero que a la vez poseen una estructura compleja que hace que cada individuo los aplique según los diversos contextos y circunstancias. ¿Qué define a una comunicación efectiva? ¿Cómo se determina el impacto del conocimiento en las diversas esferas sociales? ¿Quién determina los pasos para una negociación exitosa? Estas y muchas otras interrogantes surgen al profundizar en estos conceptos.

Por tanto, la capacidad y necesidad de relacionarse es lo que impulsa al ser humano a comunicarse con el entorno y sus pares $y$, entre otros aspectos, realizar procesos de negociación. En este ámbito, el conocimiento es sinónimo de oportunidades y la negociación marca el camino sobre las decisiones tomadas bajo circunstancias específicas.

¿Cuál es el rol de las neurociencias bajo este contexto? El prefijo «neuro» no ha pasado desapercibido. En los últimos años, una serie de ciencias y disciplinas han adoptado este concepto para ampliar el análisis sobre los comportamientos, decisiones y acciones de los individuos. Desde el marketing hasta la tecnología, las neurociencias estudian la organización y estructura del sistema nervioso, creando conexiones entre comportamientos, actividades o estímulos en la mente de las personas. Bajo este contexto, relacionar a las neurociencias con conceptos como comunicación, conocimientos y negociación, despierta el interés por comprender la complejidad de estos términos y su relación con la mente humana. Lo complejo del análisis radica en que las neurociencias integran a todas aquellas ciencias dedicadas al estudio del sistema nervioso normal y patológico (Giménez y Sánchez-Migallón, 2010), por lo que es posible plantearse la siguiente pregunta: ¿qué relación tiene el estudio del sistema nervioso con la comunicación humana, el conocimiento y la dinámica de las negociaciones? 


\section{Mente que sabe y siente}

El reconocido psicólogo Daniel Goleman (2019) se especializó en estudiar la inteligencia emocional, al ampliar en los hallazgos de otros referentes sobre la temática. Goleman resalta la existencia de dos mentes: la que sabe y la que siente; dicho en otras palabras, la mente racional y la mente emocional. La inclusión del concepto «neuro» en una serie de áreas y disciplinas desafía a las bases del concepto, que como fue mencionado anteriormente, hace referencia a las ciencias dedicadas al estudio del sistema nervioso. Sin embargo, la comunicación, el conocimiento y la negociación tienen relación directa con las neurociencias: es más, cualquier disciplina o profesión tiene un vínculo con las neurociencias. Como se ha comprobado en los últimos años, el enfoque en la educación del cerebro emocional se ha convertido en un eje diferenciador para el éxito de los seres humanos. Esta mente o cerebro también sirve como eje catalizador del cerebro que piensa, o mente racional. De esta forma, toda la gestión de las comunicaciones, absorción de conocimientos y aplicación de negociaciones conlleva una base en las relaciones que existen entre actitudes, comportamientos, estímulos y acciones, algo claramente relacionado al estudio del sistema nervioso.

Aunque el sistema tradicional se ha enfocado en la mente que piensa, la mente emocional existió mucho tiempo antes. De esta manera, un concepto tan profundo como el conocimiento se ha visto afectado por el imaginario social, relacionándolo únicamente a lo racional, cuando lo emocional juega un rol fundamental en su desarrollo. Esto se puede analizar bajo diversos contextos, pero basta reflexionar y situar a personas brillantes racionalmente que llegan a hundirse en los peligros de las pasiones desenfrenadas y de los impulsos difíciles de controlar. Por más que posean un coeficiente intelectual sobresaliente, su éxito no está garantizado.

El éxito del ser humano se mide desde distintas perspectivas, una de ellas sin duda es el coeficiente intelectual que se puede atribuir el $20 \%$, pero el otro 80 $\%$ lo determinan otras fuerzas, donde lo emocional juega un papel trascendental. Habilidades tales como motivarse, controlar los impulsos, regular el humor y evitar nublar los pensamientos forman parte de la inteligencia emocional. Estos conceptos también participan en el intelecto de los individuos, por lo que es clave resaltar que el conocimiento debe medirse desde diversas perspectivas y la comunicación, conocimiento y negociación deben contemplar a estas dos mentes como la base para la consecución de resultados exitosos. 


\section{De lo interno a lo externo}

La iniciativa parte del ser humano, pero se complementa en sociedad. De esta manera, se puede contextualizar el proceso mediante el desarrollo en el plano intrapersonal del ser humano, momento fundamental para citar a las neurociencias; al pasar a través de una integración del individuo con la sociedad, al brindar un enfoque psicosocial a la mezcla racional-emocional que despega del plano interno del ser humano e impacta en las relaciones sociales culmina en un concepto sociológico, donde el impacto trasciende las barreras relacionales y permite ser analizado bajo una macroperspectiva. Lo que resulta curioso de esta secuencia es el punto de partida: la mente humana.

La mente humana debe educarse: esa constante ampliación en conocimientos tiene que ocurrir en los pensamientos y sentimientos, lo que conlleva a desarrollar amplias habilidades en torno a la inteligencia emocional.

El psicólogo Malcolm Gladwell (2005) añade un concepto que complementa la gestión de la mente que piensa y la que siente: el cerebro intuitivo. ¿Existe tal cosa como pensar sin pensar? Gladwell presenta diversos estudios que demuestran que los «cortes finos» (la habilidad que posee el inconsciente para encontrar patrones en situaciones y comportamientos para tomar acción) juegan un papel fundamental en las relaciones de los seres humanos. Es más, él asegura que muchas de las decisiones tomadas precipitadamente pueden ser igual de buenas, o aún mejores, que las que se prolongan por diversas circunstancias.

De esta forma, la mente humana cobra otra dimensión. Para ser efectivos en los intercambios comunicacionales, ampliar conocimientos de manera consciente y gestionar efectivamente las negociaciones, se debe desarrollar un patrón repetitivo que dé espacio a una conciencia sobre el manejo de la inteligencia racional, emocional e incluso intuitiva, aquella que se maneja por lo general en el inconsciente.

Al considerar lo mencionado, es importante resaltar que la evolución ha permitido al ser humano educar a sus dos mentes. De esta forma, la especie humana está en la capacidad de conocer sus propias emociones, manejarlas mediante la autorregulación, motivarse, reconocer las emociones de los demás y gestionar sus relaciones sociales. Estos cinco elementos, claves para el desarrollo de la inteligencia de las personas, parten de lo interno hacia lo externo. Para ser partícipes de una comunicación efectiva, es necesario contemplar una evolución de estos cinco elementos, los cuales con prácticas repetitivas pueden llegar a convertirse en conductas casi innatas, que involucran al cerebro activa e intuitivamente en una gestión asertiva de ambas mentes. Este tipo de comportamientos permiten 
al ser humano adaptar los conocimientos al inconsciente adaptativo, definido por Gladwell (2005) como esa computadora gigante que rápida y silenciosamente procesa grandes cantidades de datos para hacer funcionar a los seres humanos.

\section{Tiempos precisos, negociaciones exitosas}

Para lograr negociaciones exitosas debe gestionarse efectivamente la comunicación, lo que conlleva a la aplicación de la asertividad y escucha activa a través de los diversos procesos; de la misma manera, el conocimiento juega un rol vital, pues conocerse a uno mismo y al otro amplía el horizonte de posibilidades para establecer relaciones ganar-ganar. Bajo esta premisa, es válido resaltar la importancia de los comienzos, mitades y finales.

El comienzo se encuentra en la persona, las mitades son los procesos de negociación y los finales radican en los acuerdos. Lo más complejo, como menciona Pink (2018), se encuentra en las mitades. Bajo esta complejidad es determinante gestionar las negociaciones con inteligencia, una inteligencia que debe gestionarse desde lo racional y lo emocional. Los comienzos son memorables, el individuo utiliza puntos de referencia para guiarse en el tiempo, se trata de abrir nuevas cuentas mentales; los puntos intermedios pueden jugar un doble patrón, pueden desanimar, como pueden estimular; mientras, los finales ayudan ganando energía y elevando a los individuos. Pero, ¿qué relación tienen los tiempos con las neurociencias, y específicamente con la comunicación, el conocimiento y la negociación? Como respuesta, puede afirmarse que tienen relación en todos los aspectos.

Al tomar como punto de partida que los procesos de negociación se concentran en las mitades, es importante resaltar que durante estos lapsos de tiempo se revelan efectos poderosos, según revelan estudios relacionados a la ciencia de los tiempos. Llegar al punto intermedio puede significar que los intereses de las personas se adormecen o estancan, pero a la vez, puede llegar a incitar y estimular. De esta manera se puede asociar que el inicio de los procesos de negociación se basa en las primeras percepciones, las mitades en los procesos y los finales en los acuerdos. Las mitades son claves para llegar al cierre de negocios. 


\section{Negociaciones que trascienden}

De acuerdo con Munduate y Martínez (2003) la negociación es un medio básico para conseguir de los demás aquello que se desea; pero obtener de los demás aquello que uno desea conlleva una serie de características. Para ello resulta conveniente citar al Harvard Negotiation Project: Fisher, Ury y Patton (2011) van más allá de los conceptos base de la negociación en su obra Obtenga el Sí, y proponen un modo alternativo para la gestión de las negociaciones.

Existen dos grandes áreas para el análisis de la gestión de las negociaciones: la gestión blanda y la dura. La primera de ellas se relaciona a comportamientos pasivos en las partes negociadoras; en este sentido, la negociación blanda es aquella que brinda concesiones fácilmente. Por el otro lado, la negociación dura consiste en una postura posicional, en una única alternativa para llegar a acuerdos. ¿Serán funcionales estos enfoques de gestión?

La negociación basada en principios es el enfoque propuesto por estos autores. La propuesta es ser duro y blando a la vez. Ser duro con las circunstancias y blando con las personas. Este tipo de negociación se basa en las circunstancias del lugar, la búsqueda de beneficios mutuos, normas y criterios justos. Este enfoque en gestión de la negociación contempla cuatro elementos clave: personas, intereses, opciones y criterios.

- Personas. Al mencionar a las personas, se debe establecer un marco ético y objetivo, para ello es necesario separar a las personas del problema. A pesar de que los problemas residen en la mente de las personas, este enfoque de negociación propone colocar todos los problemas sobre la mesa de discusión, dejando a las personas fuera de las percepciones y así evitar nublar los pensamientos, juzgar al otro y guiarse por estereotipos o diferencias culturales.

- Intereses. Es importante centrarse en los intereses y no en las posiciones. Esto es fundamental, pues las posiciones forman parte de la negociación dura. Cuando un individuo o grupo se enfocan en las posiciones, muestran resistencia a las alternativas.

- Alternativas. Las opciones deben ser amplias y tener diversas posibilidades que abran el marco de las negociaciones. De esta manera no se limitan las opciones y se evitan las posiciones.

- Criterios. Cumpliendo con los tres pasos anteriores, los procesos efectivos de negociación deben mantener estándares objetivos que sirvan de base para llegar a acuerdos y establecer marcos de medición de resultados. 
Este último punto marca el camino en la transición de las mitades a los finales. Cabe resaltar que los seres humanos son criaturas de fuertes emociones, con dificultades para comunicarse de forma clara, y este método de negociación busca gestionar de forma efectiva las emociones y comunicaciones de los seres humanos. Por lo tanto, las negociaciones deben contemplar un manejo óptimo de la inteligencia emocional y racional, de la mente intuitiva, de la comunicación asertiva y del conocimiento interno y externo. Ser inteligente resulta mejor que ser agresivo, una buena estrategia brinda mayores conocimientos de las situaciones, una buena comunicación va más allá de las palabras y un buen cambio empieza por quien lleva la iniciativa (Font, 2019). 


\section{Referencias}

Fisher, R., Ury, W. y Patton, B. (2011). Obtenga el Sí. Barcelona, España: Grupo Planeta.

Font, A. (2019). Las 12 Leyes de la Negociación. Ciudad de México, México: Conecta.

Giménez, J. M. y Sánchez-Migallón, S. (2010). De la Neurociencia a la Neuroética. Pamplona, España: Ediciones Universidad de Navarra, S. A.

Gladwell, M. (2005). Blink: The Power of Thinking Without Thinking. Estados Unidos de América: Little, Brown and Company.

Goleman, D. (2019). La Inteligencia Emocional. Ciudad de México, México: Penguin Random House.

Munduate, L. y Martínez, J. M. (2003). Conflicto y Negociación. Madrid, España: Pirámide.

Pink, D. (2018). ¿Cuándo? La Ciencia de Encontrar el Momento Preciso. Ciudad de México, México: Paidós Empresa. 